\title{
A Novel Tunable Triple-Band Left-Handed Metamaterial
}

\author{
Si Li, ${ }^{1}$ Atef Z. Elsherbeni, ${ }^{2}$ Wenhua Yu, ${ }^{3}$ Wenxing Li, $^{1}$ and Yunlong Mao ${ }^{1}$ \\ ${ }^{1}$ College of Communication and Information Engineering, Harbin Engineering University, Harbin, Heilongjiang 150001, China \\ ${ }^{2}$ Electrical Engineering Department, Colorado School of Mines, Golden, CO 80401, USA \\ ${ }^{3}$ 2COMU Inc., Fairfax, VA 22030, USA
}

Correspondence should be addressed to Wenxing Li; liwenxing@hrbeu.edu.cn

Received 22 June 2017; Revised 7 October 2017; Accepted 30 October 2017; Published 12 December 2017

Academic Editor: Shih Yuan Chen

Copyright (c) $2017 \mathrm{Si} \mathrm{Li} \mathrm{et} \mathrm{al.} \mathrm{This} \mathrm{is} \mathrm{an} \mathrm{open} \mathrm{access} \mathrm{article} \mathrm{distributed} \mathrm{under} \mathrm{the} \mathrm{Creative} \mathrm{Commons} \mathrm{Attribution} \mathrm{License,} \mathrm{which}$ permits unrestricted use, distribution, and reproduction in any medium, provided the original work is properly cited.

\begin{abstract}
A novel tunable triple-band left-handed metamaterial (LHM) composed of a single-loop resonator (SLR) and a variable capacitorloaded short wire pair (CL-SWP) printed on both sides of a substrate is presented in this paper. The CL-SWP-based metamaterial (MTM) is a novel single-sided LHM. It is theoretically analyzed capable of extracting tunable negative permeability and a wideband negative permittivity. We ran simulations for the CL-SWP-based MTM, the SLR-based MTM, and the proposed LHM. Together with the measured results, it is identified that this novel LHM exhibits a tunable triple-band left-handed (LH) property. With the increase of the loaded capacitance, one LH band is relatively stable, while the other two are moving towards lower frequencies with their bandwidth getting wider and narrower, respectively. The surface current density distributions indicate that the first LH band is mainly decided by the SLR, one of the rest $2 \mathrm{LH}$ bands is mainly decided by the CL-SWP, and the other one is decided by the SLR and CL-SWP together.
\end{abstract}

\section{Introduction}

Left-handed metamaterials (LHMs) are a kind of engineering material which have negative permittivity and permeability in the same frequency ranges hence exhibiting special properties such as negative refractive index, the reversal of Snell's Law, the Doppler effect, and the Vavilov-Cerenkov effect and backward waves [1]. Because of the intrinsic and narrow resonant frequency band of most LHMs, however, it cannot be used in some wide operating frequency areas $[2,3]$.

Some tunable LHMs have been proposed by using various additional materials or structures, such as the ferrites [4-9], liquid crystals [10-12], varactors [13-16], and tunable structures [17-19]. Ferrites or liquid crystals can be easily added to conventional LHMs to achieve tunability. For instance, in [8], a ferrite rod is simply added to a LHM composed of split ring resonators (SRRs) and metallic wires, and in [10], the liquid crystal is simply inserted between two omega-type resonators. Such kinds of LHMs are easy to design but have disadvantages such that ferrite-based tunability requires big magnetic bias and liquid crystal-based tunability has a finite tunable range [2]. Tunable structures can be implemented with the methods such as the displacement between layers [19] and the change of the thickness of the substrate [18]. This kind of tunability is not as convenient or flexible as the previous ones but is much easier to be implemented in practice. Varactor-loaded tunability has been used in microwave engineering applications since they can be easily integrated into microwave circuits. However, the insertion requires a proper gap region, which increases the design difficulties.

There is also another increasing requirement of multiband property for LHMs in microwave applications. Multiband LHMs could be implemented with simply arranging multiple LHM units together $[20,21]$ or with particular elements that contain multiple resonances [22-26]. Such kinds of LHMs are well designed and usually have complex metallic structures for the purpose of making the $\mu$-negative and the $\varepsilon$-negative frequency ranges overlapped.

However, tunable multiband LHMs are merely reported. In this paper, we present a novel tunable triple-band LHM. This LHM is a combination of a variable capacitor-loaded 


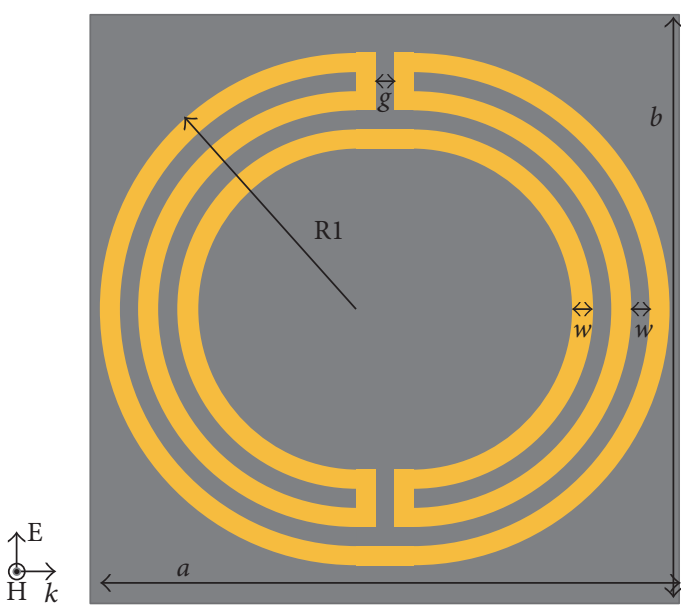

(a)

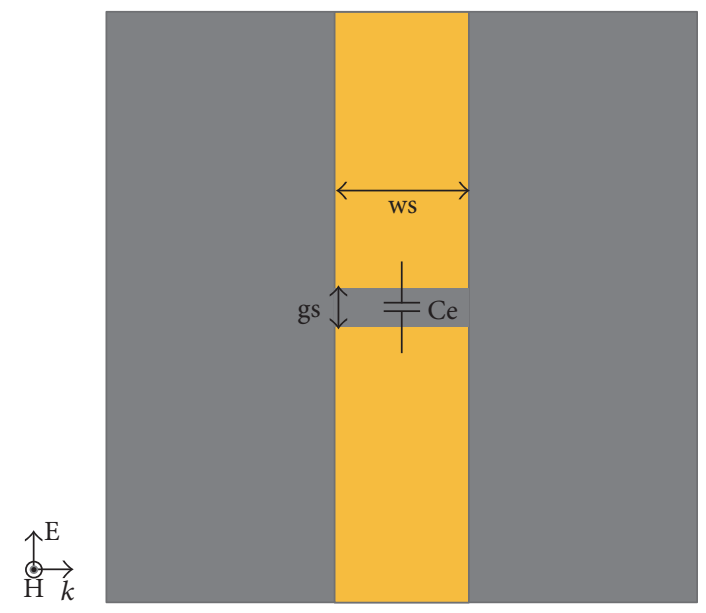

(b)

FIgURE 1: The geometry of one unit of the (a) SLR-based MTM and (b) CL-SWP-based MTM.

short wire pair (CL-SWP) and circular single-loop resonator (SLR) structures printed on both sides of a substrate. The CLSWP-based metamaterial (MTM) is single-sided, and it is a novel kind LHM. We theoretically analyzed the CL-SWPbased MTM. The SLR-based MTM [25, 27, 28] is also single-sided and is capable of extracting dual-band negative permeability. In our design, we print the circular SLR on the other side of the CL-SWP-based MTM to build a novel LHM. Additionally, we also studied the connections among the CL-SWP, the SLR, and the novel tunable triple-band LHMs with simulations and the help of "S parameter retrieval" method [29].

\section{Model and Theoretical Analysis}

The geometry of the SLR-based MTM and the CL-SWPbased MTM is shown in Figures 1(a) and 1(b), respectively, where the yellow lines represent metallic wires with a thickness of $0.018 \mathrm{~mm}$. The incidence is as illustrated, where $k$ is the incident direction and $\mathrm{E}$ and $\mathrm{H}$ refer to the directions of the electric field and magnetic field, respectively. Both the structures are printed on a $15 \mathrm{~mm} \times 15 \mathrm{~mm} \times 0.8 \mathrm{~mm}$ Rogers 6006 substrate whose relative permittivity is 6.15 . Here, $a=15 \mathrm{~mm}, b=15 \mathrm{~mm}, \mathrm{R} 1=6.6 \mathrm{~mm}, \mathrm{w}=0.45 \mathrm{~mm}$, $\mathrm{g}=0.25 \mathrm{~mm}$, ws $=4 \mathrm{~mm}$, and $\mathrm{gs}=1 \mathrm{~mm}$.

The SLR-based MTM have previously been discussed and identified capable of extracting multiband negative permeability [25, 27, 28]. In this paper, we designed a circular SLR for the purpose of restraining electric coupling in the design of the tunable triple-band LHM.

Since the CL-SWP-based MTM is a novel structure, it is important to explain why it is capable of extracting tunable negative permeability and a wide-band negative permeability. Depending on the theoretical analysis of the SRRs proposed in $[30,31]$, magnetic resonance is motivated by the electromotive force around the circumference of the split rings. Considering an infinite array of such SRRs arranged in three orthogonal directions with a spatial period of $d$ and an incident magnetic field polarized along the $y$ direction, that is, perpendicular to the SRRs, there would be an electromotive force and an induced current $I$ along the rings, satisfying the following:

$$
\mathrm{EMF}=j \omega \pi r^{2} \mu_{0} H_{0}=\frac{R I+I}{(-j \omega C)+(-j \omega L) I+(-j \omega F L) I},
$$

where $H_{0}$ is the external magnetic field, $R, L$, and $C$ are the parasitic resistance, inductance, and capacitance of each ring, respectively, and $F L$ is the mutual inductance between different rings. With $L=\pi r^{2} \mu_{0} / d$, and $F=\pi r^{2} / d^{2}$, the current $I$ is given as follows:

$$
\begin{aligned}
I & =\frac{-j \omega \pi r^{2} \mu_{0} H_{0}}{j \omega \pi r^{2} \mu_{0}\left[1-\left(\pi r^{2} / d^{2}\right)\right]-R+(1 / j \omega C)} \\
& =\frac{-d H_{0}}{\left(1-F-\left(1 / \omega^{2} L C\right)\right)+(R j / \omega L)} .
\end{aligned}
$$

Based on the magnetic moment per unit volume, $M=\pi$ $r^{2} I / d^{3}$ and $\mu_{\text {eff }}=\left(B / \mu_{0}\right) /\left(B / \mu_{0}-M\right)$, where $B$ is the corresponding external magnetic flux; then we can obtain the final effective permeability as follows:

$$
\begin{aligned}
\mu_{\mathrm{eff}}= & 1-\frac{F\left(1-\left(1 / \omega^{2} L C\right)\right)}{\left(1-\left(1 / \omega^{2} L C\right)\right)^{2}+\left(R^{2} /(\omega L)^{2}\right)} \\
& +j \frac{R / \omega L}{\left(1-\left(1 / \omega^{2} L C\right)\right)^{2}+\left(R^{2} /(\omega L)^{2}\right)} .
\end{aligned}
$$

From the analysis above, it is obvious that the effective permeability is completely irrelevant to the shape of the magnetic resonators. The real part of $\mu_{\mathrm{eff}}$ can be negative with proper selections of $F, R, L$, and $C$, while the imaginary part will always be positive.

For the CL-SWP-based MTM, the loaded capacitance reversed the current flux on both open ends in the middle. Then the external magnetic field will motive the magnetic resonance around the middle gap. For this given structure, " $L$ " and " $R$ " are relatively unchangeable, while " $C$ " is mainly 


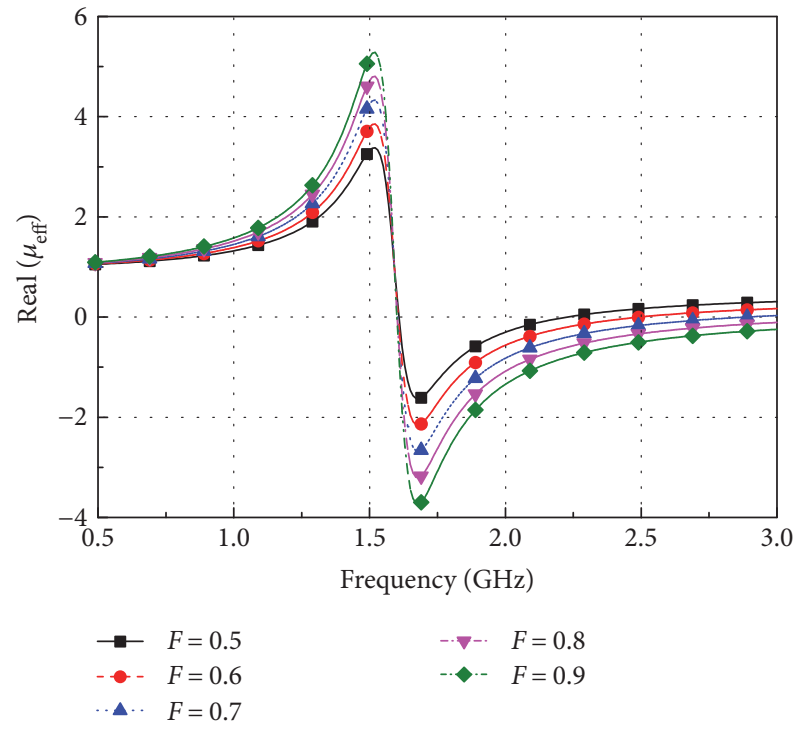

Figure 2: The real part of the effective permeability when $R=1 \mathrm{ohm}$, $L=1 \mathrm{nH}$, and $C=10 \mathrm{pF}$.

decided by the loaded capacitance and " $F$ " may be affected by other electromagnetic interference as we will discuss in the following sections. Hence, with proper values of " $C$ " or " $F$," the CL-SWP-based MTM is capable of extracting tunable negative permeability as we can draw from (3). If we have $R=1 \mathrm{ohm}, L=1 \mathrm{nH}$, and $C=10 \mathrm{pF}$, then the change of the real part of $\mu_{\text {eff }}$ with $F$ changing from 0.5 to 0.9 is displayed in Figure 2, where "Real $\left(\mu_{\text {eff }}\right)$ " represents the real part of the effective permeability. As can be drawn from Figure 2, the increase of $F$ not only increased the maximum absolute value of the real part of $\mu$ but also widened the $\mu$ negative bandwidth.

On the other hand, the connected short wires are serving as arrayed metallic wires; hence, they are capable of extracting negative permittivity, as analyzed in [32]. However, this connection also brings an electric resonance. Since the domain size is much smaller than the wavelength of the incidence, the external electric field across a unit can be approximated as $U=E_{\mathrm{z}} b=R I+(-j \omega L) I+I /(-j \omega C)$, where $E_{\mathrm{z}}$ is the external electric field per length. Then, the volume current density $J_{\mathrm{v}}$ in each unit can be homogenized as follows:

$$
J_{\mathrm{v}}=\frac{I}{a^{2}}=\frac{j \omega /(a L)}{\omega^{2}-(1 /(L C))+(j \omega R / L)} E_{\mathrm{z}}=\sigma^{\prime} E_{\mathrm{z}} .
$$

While in [31],

$$
\varepsilon_{\text {eff }}=1+j \frac{\sigma^{\prime}}{\omega \varepsilon_{0}}
$$

Hence,

$$
\varepsilon_{\text {eff }}=1-\frac{\left[\left(\omega^{2}-(1 / L C)\right) /\left(a L \varepsilon_{0}\right)\right]-\left(j \omega R / a L^{2} \varepsilon_{0}\right)}{\left[\omega^{2}-(1 /(L C))\right]^{2}+(\omega R / L)^{2}} .
$$

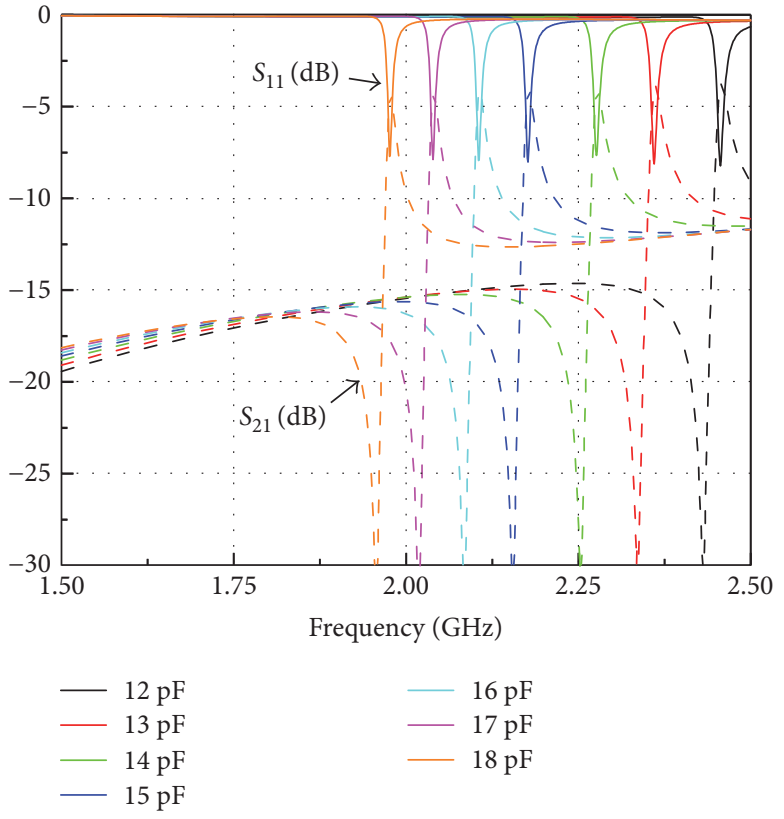

FIgURE 3: Simulated S parameters for the CL-SWP-based MTM with the loaded capacitance ranging from $12 \mathrm{pF}$ to $18 \mathrm{pF}$. The solid lines represent $S_{11}(\mathrm{~dB})$, while the dashed lines represent $\mathrm{S}_{21}(\mathrm{~dB})$.

From (6), at the resonant frequency, the real part of the effective permittivity is 1 . With the increase of the frequency, the real part of the effective permittivity is getting smaller and it may be negative at frequencies higher than the resonant frequency. Therefore, the effective permittivity of CL-SWP is a gaming result of its plasma frequency and the electric resonance. It can be negative in a wide frequency band if the electric resonance is not strong enough.

\section{Simulations and Analysis}

Based on the foregoing analysis, we print the circular SLR structure on the other side of the CL-SWP-based LHM, for the purpose of building a novel tunable triple-band LHM. In this section, we ran simulations for the CL-SWP-based MTM, the circular SLR-based MTM, and the combined LHM, respectively, with full-wave simulator HFSS. The loaded capacitance are chosen ranging from $12 \mathrm{pF}$ to $18 \mathrm{pF}$, as an example. The effective parameters are retrieved through the "S parameter retrieval" method.

3.1. CL-SWP-Based MTM. The simulated S parameters for the CL-SWP-based MTM with the loaded capacitance ranging from $12 \mathrm{pF}$ to $18 \mathrm{pF}$ is displayed in Figure 3, where the solid lines represent $S_{11}(\mathrm{~dB})$, while the dashed lines represent $S_{21}(\mathrm{~dB})$. The corresponding retrieved effective parameters are exhibited in Figures 4(a)-4(d), where all the solid lines represent the real part while the dashed lines represent the imaginary part of the parameters. The same rule is applied in the following figures for effective parameters.

In Figure 3, the resonant frequency is shifting from $2.456 \mathrm{GHz}$ to $1.976 \mathrm{GHz}$, with the loaded capacitance ranging 


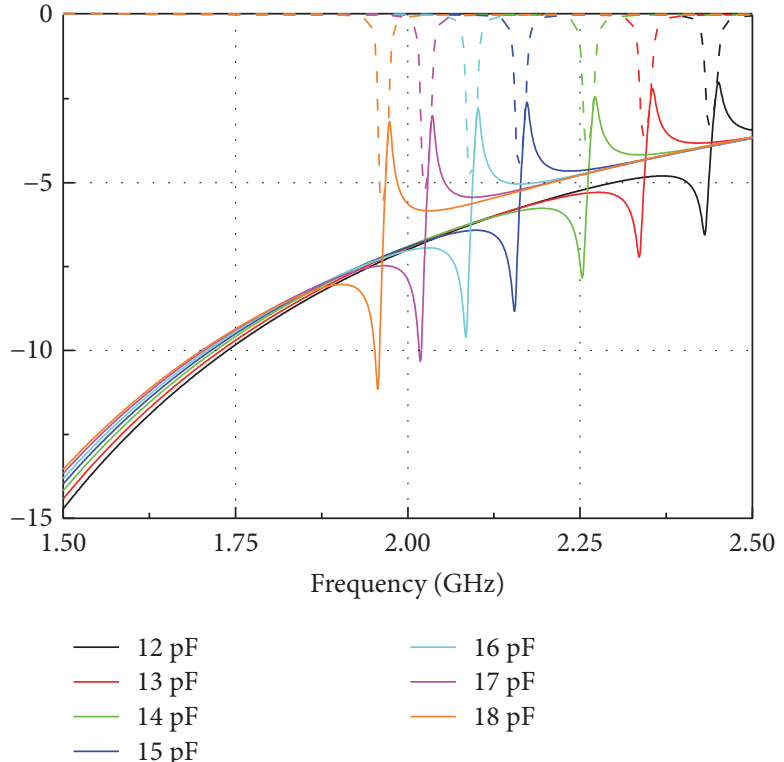

(a)

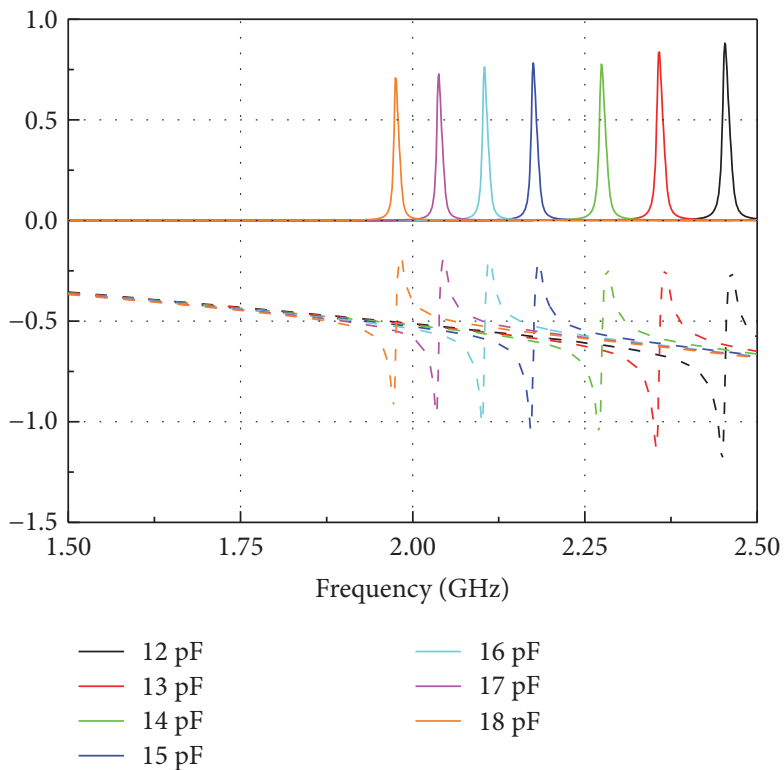

(c)

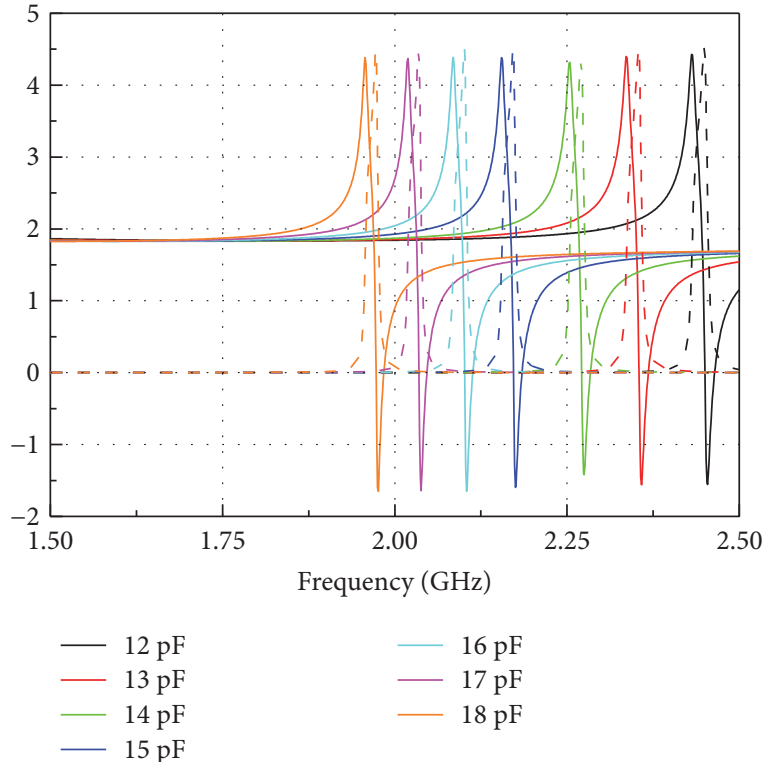

(b)

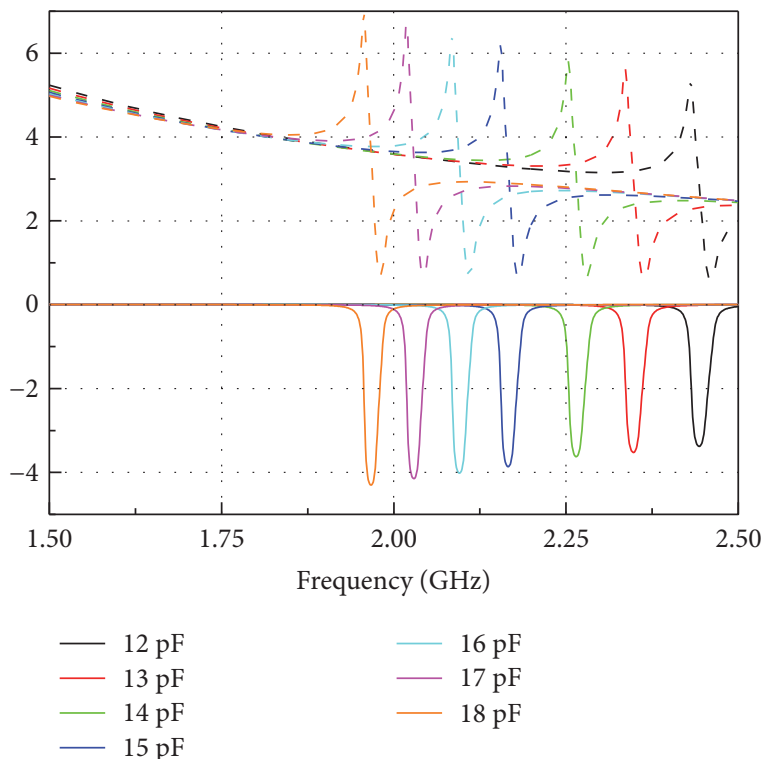

(d)

FIGURE 4: Retrieved effective (a) permittivity, (b) permeability, (c) impedance, and (d) refractive index with the loaded capacitance ranging from $12 \mathrm{pF}$ to $18 \mathrm{pF}$. The solid lines represent the real part, and the dashed lines represent the imaginary part of the parameters, respectively.

from $12 \mathrm{pF}$ to $18 \mathrm{pF}$. However, the transmission character is not good. In Figure 4(a), negative permittivity is achieved at frequencies lower than the plasma frequency [33] of the arrayed wires. With the change of the loaded capacitance, electric resonances can be observed; however, they are not strong enough to make the permittivity positive. In Figure 4(b), negative permeability is achieved, and it is tunable with the change of the loaded capacitance. Negative refractive index is also observed tunable with the change of the loaded capacitance, as illustrated in Figure 4(d). Hence, the CL-SWP-based MTM exhibit a tunable left-handed (LH) property.
3.2. SLR-Based MTM. Figure 5(a) displays the simulated $S$ parameter of the SLR-based MTM, and Figure 5(b) displays the real part of the corresponding retrieved effective parameters. In Figure 5(a), the solid black line represents $S_{11}$, while the dashed red line represents $S_{21}$. There are two transmission valleys and the corresponding minimum $S_{21}$ are observed at $1.759 \mathrm{GHz}$ and $2.235 \mathrm{GHz}$, respectively, which indicates that around these frequencies, the MTM may exhibit a single-negative (SNG) property. Accordingly, in Figure 5(b), negative permeability can be observed from $1.743 \mathrm{GHz}$ to $1.816 \mathrm{GHz}$ and $2.208 \mathrm{GHz}$ to $2.305 \mathrm{GHz}$, while the permittivity are all positive at these frequencies. The 

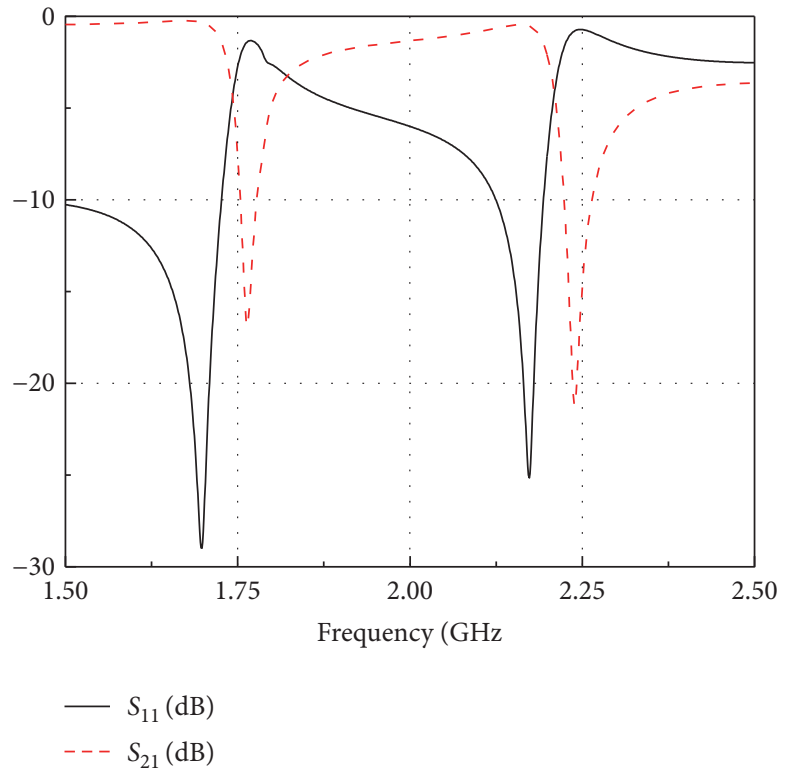

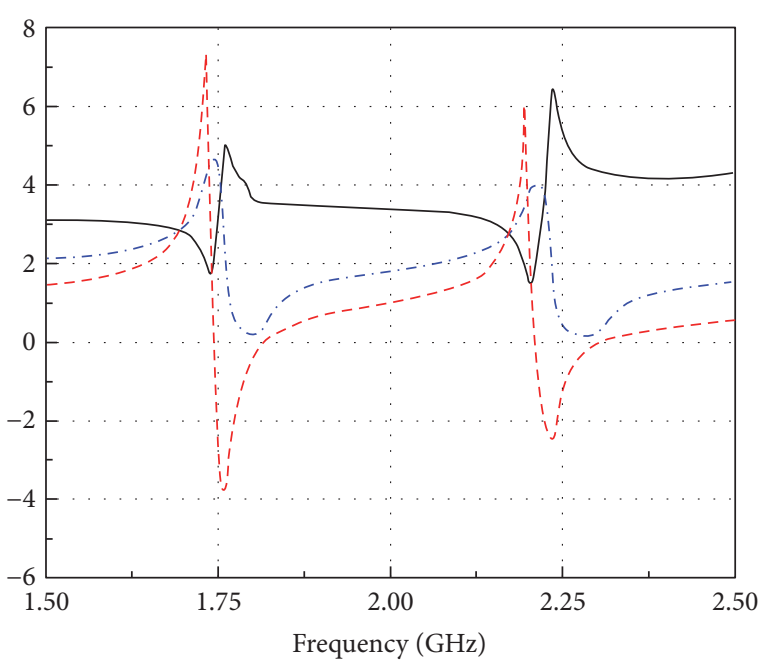

$-\operatorname{Real}\left(\varepsilon_{\text {eff }}\right)$
$\ldots-\operatorname{Real}\left(\mu_{\text {eff }}\right)$
$\ldots-\operatorname{Real}(n)$

(a)

(b)

Figure 5: Simulated (a) $S$ parameters and (b) the corresponding retrieved real part of the effective permittivity (real ( $\left.\varepsilon_{\text {eff }}\right)$, black solid line), permeability (real $\left(\mu_{\text {eff }}\right)$, red dashed-dotted line), and refractive index (real $(n)$, blue dashed-dotted line).

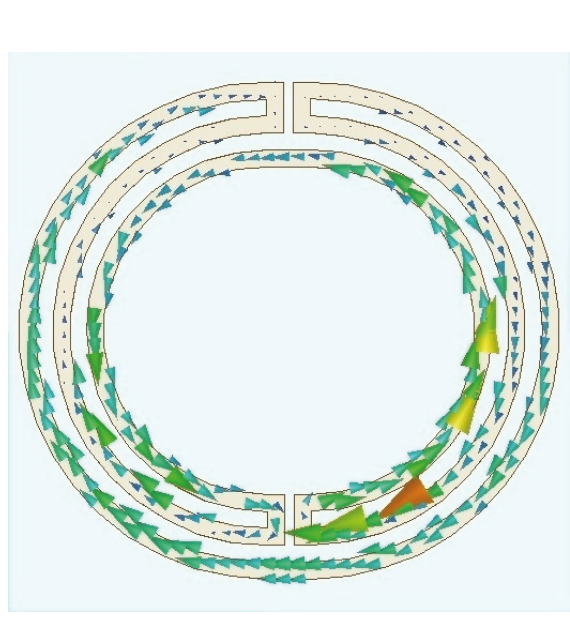

(a)

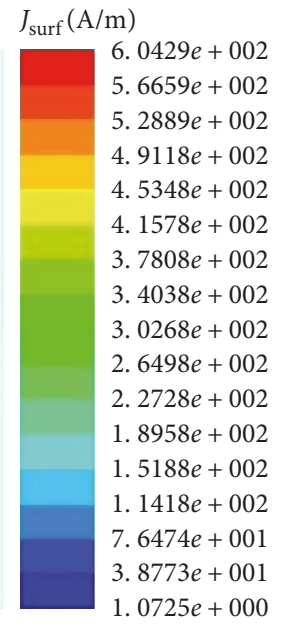

Figure 6: Surface current density at (a) $f_{1}=1.759 \mathrm{GHz}$ and (b) $f_{2}=2.235 \mathrm{GHz}$. minimum value of permeability is observed at $1.759 \mathrm{GHz}$ in the first SNG frequency range and is at $2.235 \mathrm{GHz}$ in the second SNG frequency range. Therefore, this SLR-based MTM is a dual-band $\mu$-negative MTM.

We also displayed the surface current density at these 2 resonant frequencies, $f_{1}=1.759 \mathrm{GHz}$ and $f_{2}=2.235 \mathrm{GHz}$, respectively, in Figures $6(\mathrm{a})$ and $6(\mathrm{~b})$. The results imply that $f_{1}$ is related to the bottom gap, while $f_{2}$ is related to the top gap.

3.3. Tunable Triple-Band LHM. Finally, we come to the simulations for the combined novel tunable triple-band LHM.
The simulated $S$ parameters with the change of loaded capacitance are displayed in Figure 7, where the solid lines represent $S_{11}$ and the dashed lines represent $S_{21}$.

In Figure 7 , there are 3 passband $\left(S_{11}<-10 \mathrm{~dB}\right)$. Despite the increase of the loaded capacitance, the first passband is almost unchanged and its resonant frequency is approximately $1.75 \mathrm{GHz}$. The second passband is moving towards lower frequencies, and its minimum $S_{11}$ is getting larger and its bandwidth is getting narrower with the increase of the loaded capacitance. For the third passband, when the loaded capacitance is $12 \mathrm{pF}$ and $13 \mathrm{pF}$, its resonant frequency is $2.407 \mathrm{GHz}$ and $2.336 \mathrm{GHz}$, respectively. 


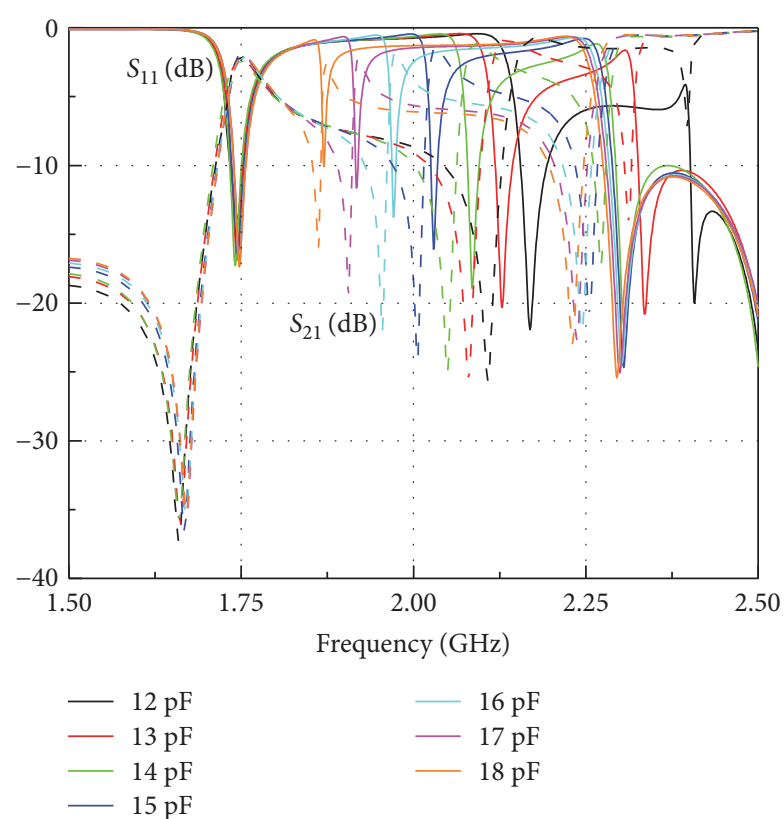

FIGURE 7: Simulated $S$ parameters with the loaded capacitance ranging from $12 \mathrm{pF}$ to $18 \mathrm{pF}$.

For other capacitance, its resonant frequency is approximately $2.3 \mathrm{GHz}$.

The retrieved effective parameters are shown in Figures $8(a)-8(d)$. In Figure $8(a)$, the real part of permittivity is negative at frequencies lower than $2.45 \mathrm{GHz}$. In Figure 8 (b), negative permeability is observed tunable at 3 different frequency ranges. These corresponding $\mu$-negative frequency ranges at different loaded capacitance are also listed in Table 1 which provides us with a more direct vision of their tunability. The " 1 st $\mu \mathrm{NG}$," " 2 nd $\mu \mathrm{NG}$," and " $3 \mathrm{rd}$ $\mu \mathrm{NG}$ " represent the $\mu$-negative frequency ranges, "BW" represent the bandwidth of the frequency range in the previous column in Table 1, and they are all measured with "GHz." The first $\mu$-negative frequency range is relatively stable around $1.7 \mathrm{GHz}$, and its bandwidth is around $0.08 \mathrm{GHz}$. The second $\mu$-negative range is moving towards lower frequencies, and its bandwidth is getting narrower with the increase of the loaded capacitance. The third $\mu$-negative range is also moving towards lower frequencies with the increase of the loaded capacitance, but its bandwidth is getting wider until the loaded capacitance is larger than $16 \mathrm{pF}$. The refractive index show a corresponding tunable tendency, as illustrated in Figure 8(d).

For a better comparison to explore the contributions the CL-SWP and the SLR made to the novel tunable triple-band LHM, we displayed the surface current density distributions at the 3 resonant frequencies: $1.745 \mathrm{GHz}$, $2.169 \mathrm{GHz}$, and $2.407 \mathrm{GHz}$ when the loaded capacitance is $12 \mathrm{pF}$ in Figures 9(a), 9(c), and 9(e), respectively, and $1.749 \mathrm{GHz}, 2.03 \mathrm{GHz}$, and $2.306 \mathrm{GHz}$ when the loaded capacitance is $15 \mathrm{pF}$ in Figures 9(b), 9(d), and 9(f), respectively. The current distribution in Figures 9(a) and 9(b) are all almost focused on the bottom gap of the SLR, which are similar to that displayed in Figure 6(a); hence, the first LH band is mainly decided by the first magnetic resonance of the SLR. In Figure 9(c), surface currents are observed focused on the mid gap of the CL-SWP, and the top gap of the SLR, while in Figure 9(e), they are mainly distributed on the mid gap of the CL-SWP. Hence, when the loaded capacitance is $12 \mathrm{pF}$, the second $\mathrm{LH}$ band is decided by the CL-SWP and the SLR together, while the third LH band is mainly decided by the CL-SWP. In Figure 9(d), surface currents are observed almost all around the mid gap of the CL-SWP, while in Figure 9(f), they are observed both around the gaps of CL-SWP and the SLR. Hence, when the loaded capacitance is $15 \mathrm{pF}$, the second LH band is mainly decided by the CL-SWP, while the third LH band is decided by the CL-SWP and the SLR together. Therefore, we can now come to a conclusion that the CL-SWP-related LH band has wider tuning frequency ranges than the SLR-related ones. Except for that, the existence of the magnetic resonances of the SLR enhanced the mutual coupling coefficient " $F$ " of the CL-SWP, and hence the LH performance of the CL-SWPrelated LH band in the novel LHM is much better than that displayed in Figure 4.

Finally, we use the standard rectangular waveguide method to experimentally characterize the effective parameters of the fabricated metamaterial [34]. The measurement setup is displayed in Figure 10(a), where a standard BJ22 waveguide is available at $1.72 \mathrm{GHz}$ to $2.61 \mathrm{GHz}$. A singlelayer sample consisting of 7 pieces of $3 \times 1$ units is used. The $S$ parameters are measured using Agilent's E8361C vector network analyzer. The loaded capacitance is $15 \mathrm{pF}$ for simplicity, as an example. The retrieved effective parameters from the simulations and the measurements, tagged with "Sim." and "Mea.," respectively, are displayed in Figure 10(b) with the frequencies ranging from $1.72 \mathrm{GHz}$ to $2.5 \mathrm{GHz}$.

In Figure 10(b), there are two frequency bands where the proposed sample exhibits double-negative properties. Compared to the simulated results, it is figured out that they correspond to the second and the third LH bands that are illustrated in Figure 8 while a little shifted to lower frequencies. The first LH band is not observed, as it is beyond the operating frequencies of the waveguide.

\section{Conclusion}

In this paper, we present a novel tunable triple-band LHM. This LHM is composed of a pair of short wires connected through a variable capacitor printed on one side of the substrate and a circular single-loop resonator printed on the other side of the substrate. The CL-SWP-based MTM have been theoretically analyzed. Simulations are operated with HFSS, for the CL-SWP-based MTM, the SLR-based MTM, and their combined novel LHM, respectively. Together with the measured results, the novel LHM is identified to have 3 tunable LH bands. It is also pointed out that the first LH band is mainly decided by the first magnetic resonance of SLR, and one of the rest two LH bands is mainly decided by the CL-SWP, and the other one is decided by the SLR and the CL-SWP together. 

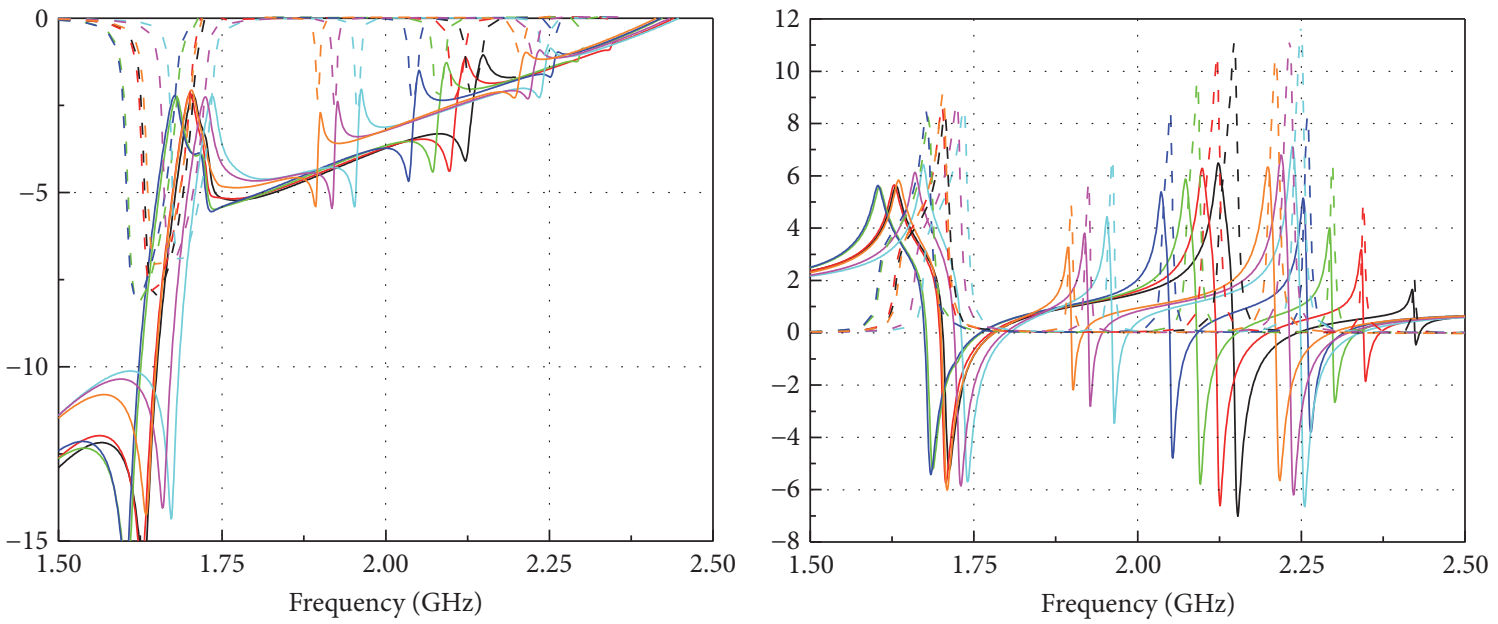

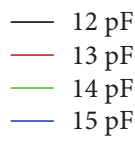

$16 \mathrm{pF}$

$-17 \mathrm{pF}$

$18 \mathrm{pF}$

(a)

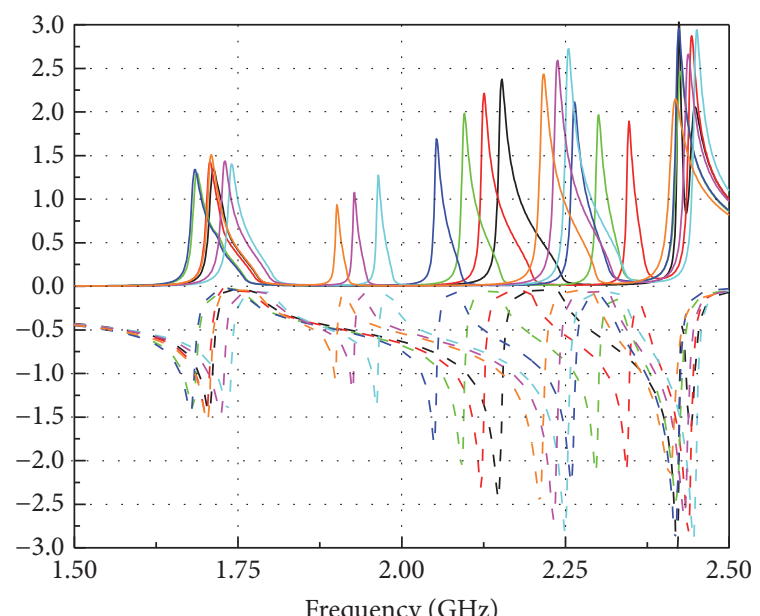

$\begin{array}{rl}-12 \mathrm{pF} & -16 \mathrm{pF} \\ -13 \mathrm{pF} & 17 \mathrm{pF} \\ -14 \mathrm{pF} & -18 \mathrm{pF} \\ -15 \mathrm{pF} & \end{array}$

(c)

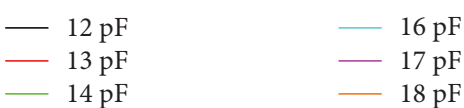

(b)

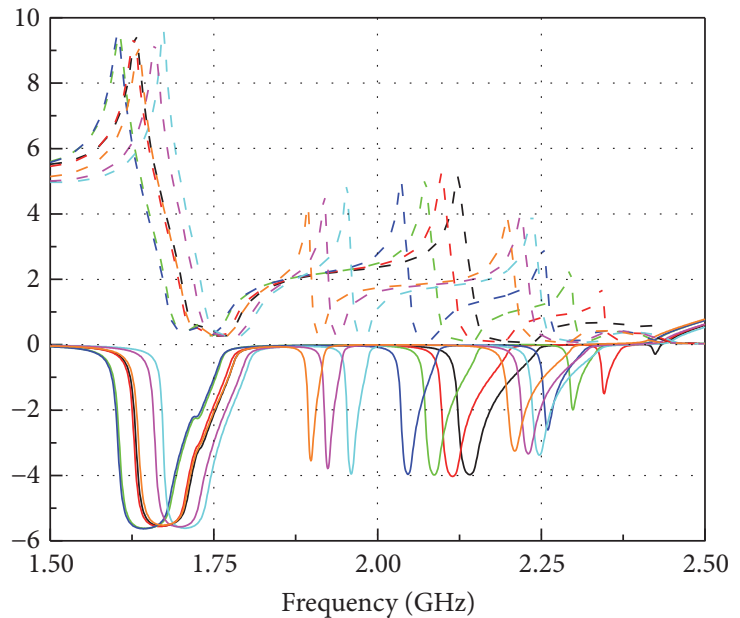

$-12 \mathrm{pF}$
$-\quad 13 \mathrm{pF}$
$-\quad 14 \mathrm{pF}$
$-15 \mathrm{pF}$

$16 \mathrm{pF}$

$-17 \mathrm{pF}$

$-18 \mathrm{pF}$

(d)

FIgURE 8: Retrieved (a) permittivity, (b) permeability, (c) impedance, and (d) refractive index with the loaded capacitance ranging from $12 \mathrm{pF}$ to $18 \mathrm{pF}$. The solid lines represent the real part and the dashed lines represent the imaginary part of the parameters.

TABLE 1: Corresponding $\mu$-negative frequency ranges $(\mathrm{GHz})$ at different loaded capacitance.

\begin{tabular}{lccccc}
\hline Ce $(\mathrm{pF})$ & 1 st $\mu$ NG & BW & 2nd $\mu$ NG & BW & 3rd $\mu$ NG \\
\hline 12 & $1.702 \sim 1.782$ & 0.08 & $2.145 \sim 2.245$ & 0.1 & $2.423 \sim 2.431$ \\
13 & $1.697 \sim 1.776$ & 0.079 & $2.119 \sim 2.2$ & 0.081 & $2.345 \sim 2.37$ \\
14 & $1.677 \sim 1.762$ & 0.085 & $2.09 \sim 2.152$ & 0.062 & $2.297 \sim 2.336$ \\
15 & $1.674 \sim 1.759$ & 0.085 & $2.048 \sim 2.091$ & 0.043 & $2.259 \sim 2.315$ \\
16 & $1.73 \sim 1.804$ & 0.074 & $1.961 \sim 1.986$ & 0.025 & $2.248 \sim 2.34$ \\
17 & $1.719 \sim 1.797$ & 0.078 & $1.924 \sim 1.946$ & 0.022 & $2.231 \sim 2.322$ \\
18 & $1.699 \sim 1.781$ & 0.082 & $1.898 \sim 1.917$ & 0.019 & 0.039 \\
\hline
\end{tabular}




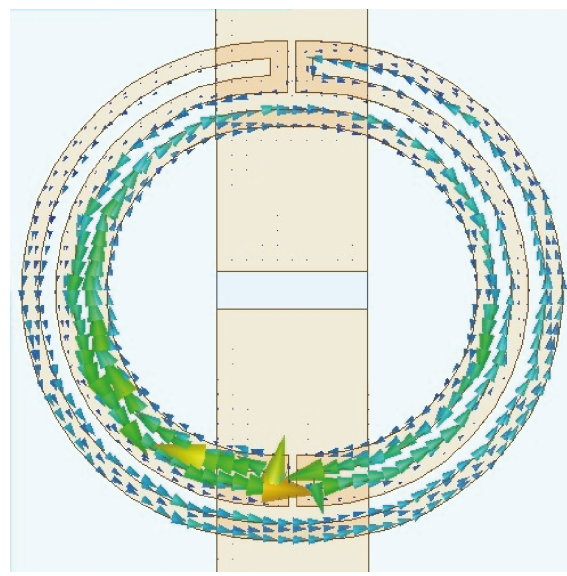

(a)

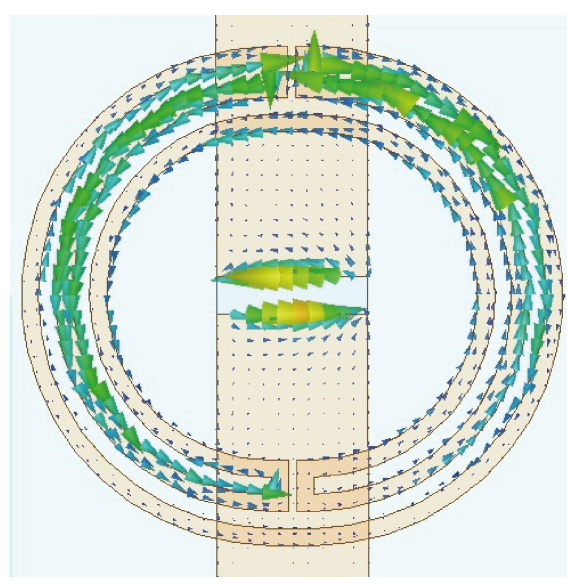

(c)

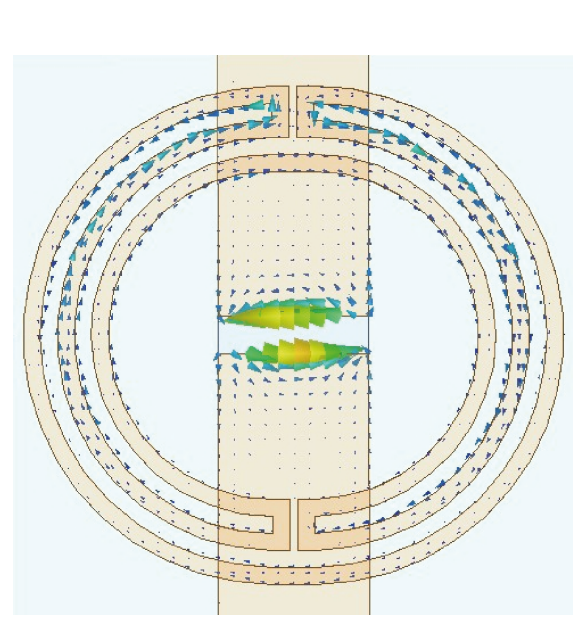

(e)

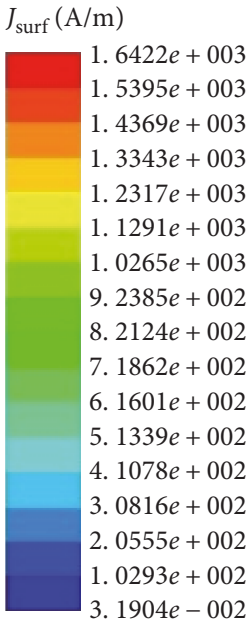

$J_{\text {surf }}(\mathrm{A} / \mathrm{m})$
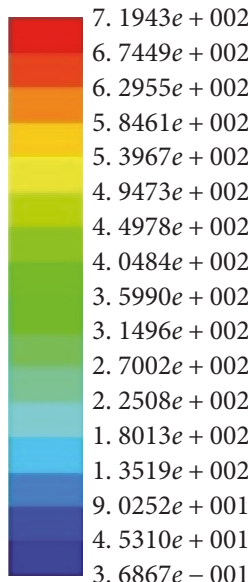

. $6867 e-001$

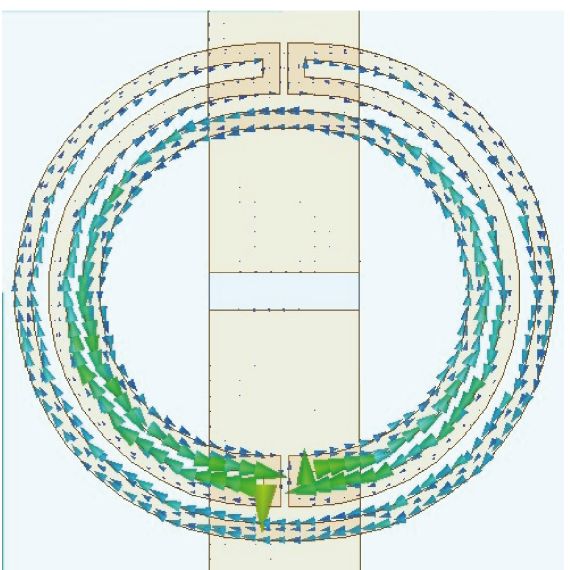

(b)
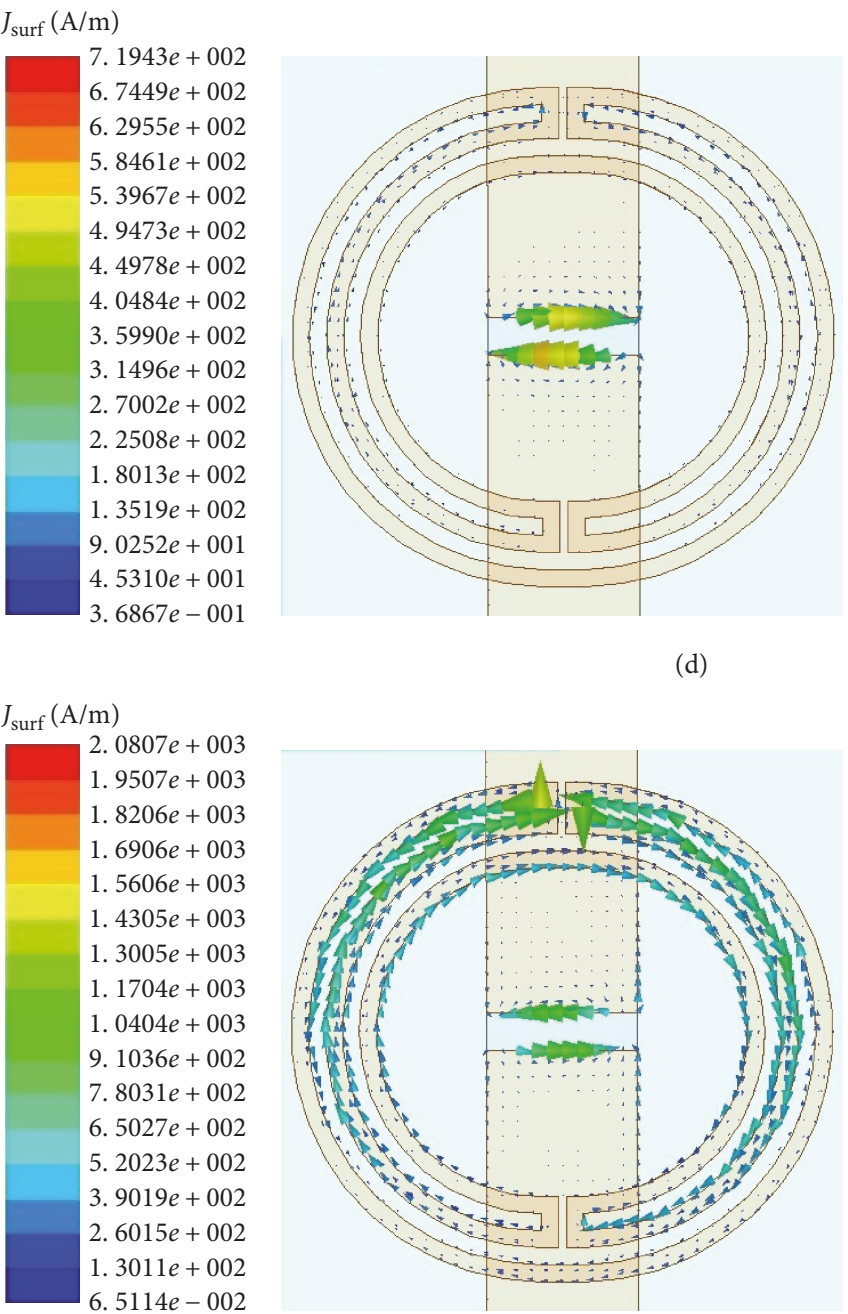

(d)

6. $5114 e-002$

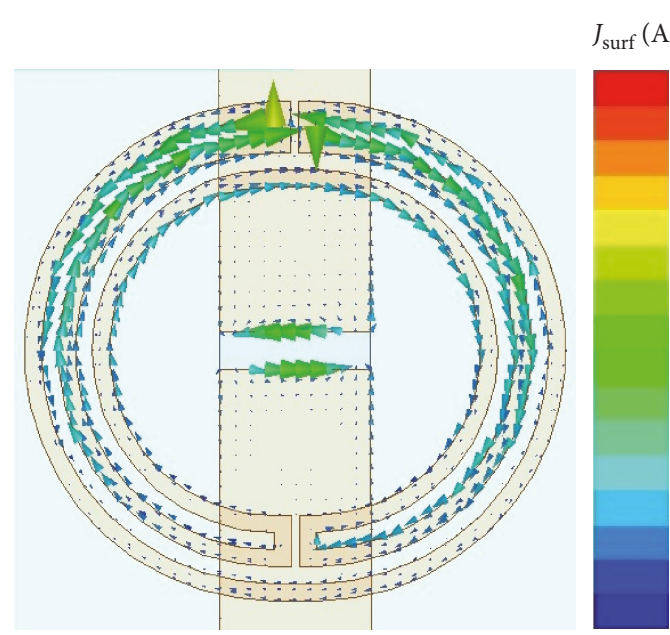

(f)
$J_{\text {surf }}(\mathrm{A} / \mathrm{m})$

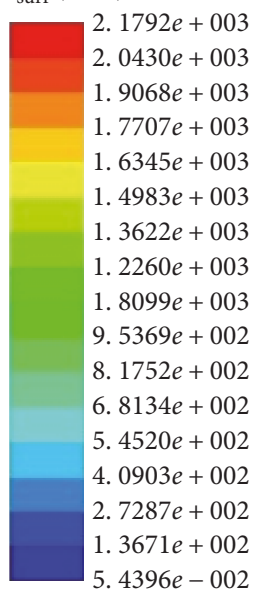

$J_{\text {surf }}(\mathrm{A} / \mathrm{m})$

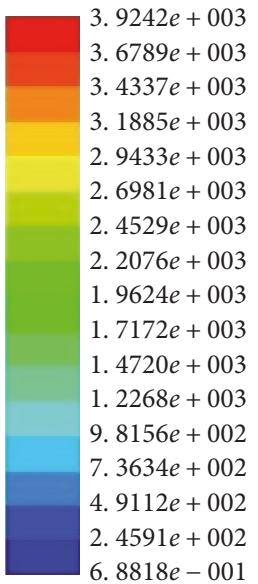

$6.8818 e-001$

\section{urf $(\mathrm{A} / \mathrm{m})$}

1. $3791 e+003$
1. $2872 e+003$
1. $1953 e+003$
1. $1034 e+003$
1. $0115 e+003$
9. $1955 e+002$
8. $2765 e+002$
7. $3574 e+002$
6. $4384 e+002$
5. $5194 e+002$
4. $6003 e+002$
3. $6813 e+002$
2. $7623 e+002$
1. $8432 e+002$
9. $2420 e+001$
5. $1704 e-001$

5. $1704 e-001$

Figure 9: Surface current density distribution at the 3 resonant frequencies: (a) $1.745 \mathrm{GHz}$, (c) $2.169 \mathrm{GHz}$, and (e) $2.407 \mathrm{GHz}$ when the loaded capacitance is $12 \mathrm{pF}$ and (b) $1.747 \mathrm{GHz}$, (d) $2.03 \mathrm{GHz}$, and (f) $2.306 \mathrm{GHz}$ when the loaded capacitance is $15 \mathrm{pF}$. 

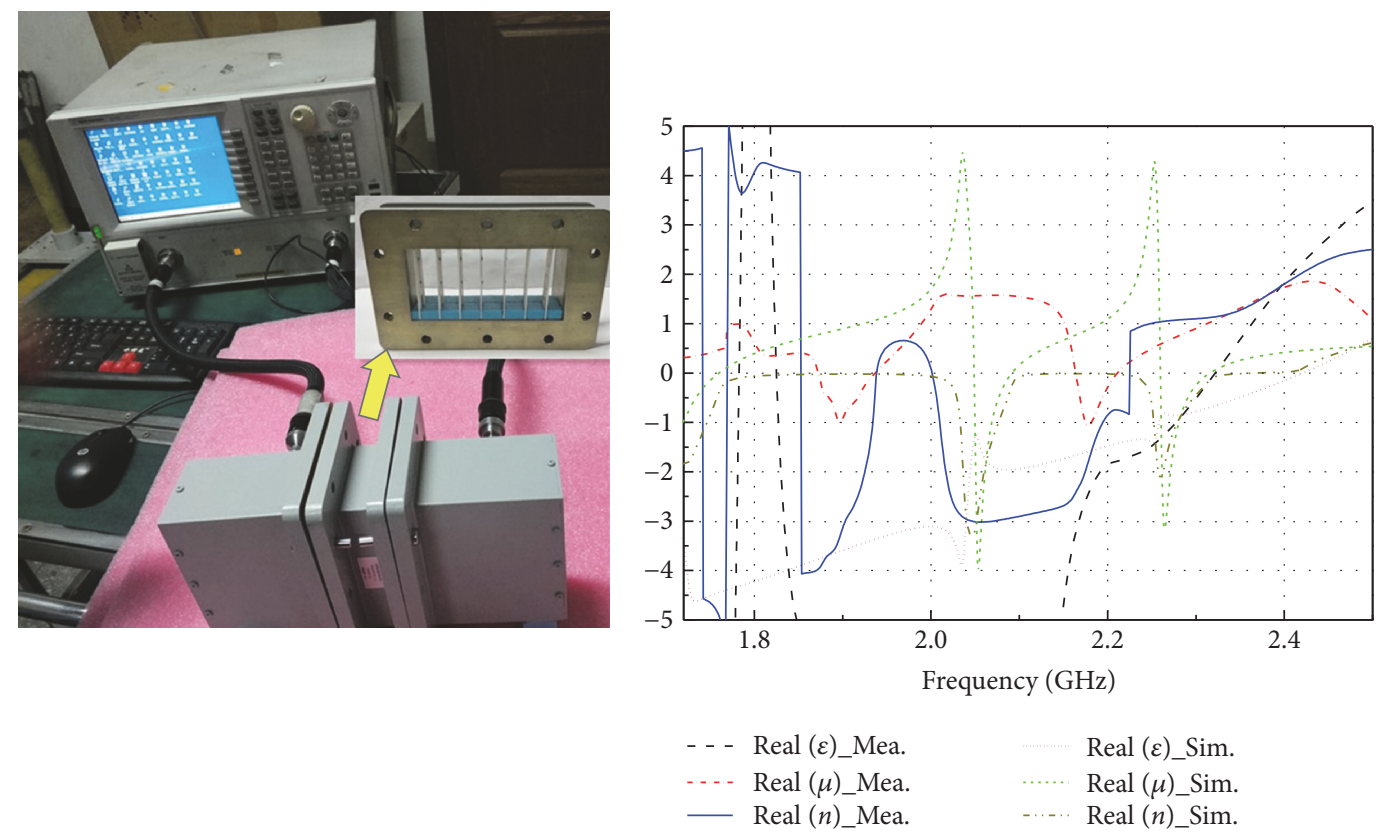

(a)

(b)

Figure 10: (a) Measurement setup and (b) the retrieved effective parameters.

\section{Conflicts of Interest}

The authors declare that there is no conflict of interests regarding the publication of this paper.

\section{Acknowledgments}

This paper was supported by National Defense "973" Basic Research Development Program of China (no. 6131380101), by preresearch fund of the 12th Five-Year Plan (nos. 4010403020102 and 4010103020103), and the Fundamental Research Funds for the Central Universities (nos. HEUCFD1433 and HEUCF1508).

\section{References}

[1] A. Lai, T. Itoh, and C. Caloz, "Composite right/left-handed transmission line metamaterials," IEEE Microwave Magazine, vol. 5, no. 3, pp. 34-50, 2004.

[2] J. Zhong, Y. Huang, G. Wen, H. Sun, and W. Zhu, "The design and applications of tunable metamaterials," Procedia Engineering, vol. 29, no. 29, pp. 802-807, 2012.

[3] N. I. Zheludev and Y. S. Kivshar, "From metamaterials to metadevices," Nature Materials, vol. 11, no. 11, pp. 917-924, 2012.

[4] B. Du, Z. Xu, J. Wang, and S. Xia, "Magnetically tunable ferrite-dielectric left-handed metamaterial," Progress In Electromagnetics Research C, vol. 66, pp. 21-28, 2016.

[5] Y. Yong-Jun, H. Yong-Jun, W. Guang-Jun, Z. Jing-Ping, S. Hai-Bin, and O. Gordon, "Tunable broadband metamaterial absorber consisting of ferrite slabs and a copper wire," Chinese Physics B, vol. 21, no. 3, article 038501, 2012.

[6] H. U. Zhen-Yan, "Novel tunable ferrite-wire chessboard structure left-handed metamaterial," Chinese Journal of Radio Science, vol. 24, no. 5, pp. 799-792, 2009.
[7] P. He, J. Gao, Y. Chen, P. V. Parimi, C. Vittoria, and V. G. Harris, "Q-band tunable negative refractive index metamaterial using Sc-doped BaM hexaferrite," Journal of Physics D Applied Physics, vol. 42, no. 15, article 155005, 2009.

[8] L. Kang, Q. Zhao, H. Zhao, and J. Zhou, "Ferrite-based magnetically tunable left-handed metamaterial composed of SRRs and wires," Optics Express, vol. 16, no. 22, pp. 17269 17275, 2008.

[9] Y. Poo, R. X. Wu, G. H. He, and P. Chen, "Experimental verification of a tunable left-handed material by bias magnetic fields," Applied Physics Letters, vol. 96, no. 16, pp. 161902 161902-3, 2010.

[10] F. Zhang, L. Kang, Q. Zhao, J. Zhou, X. Zhao, and D. Lippens, "Magnetically tunable left handed metamaterials by liquid crystal orientation," Optics Express, vol. 17, no. 6, pp. 4360-6, 2009.

[11] D. Shrekenhamer, W.-C. Chen, and W. J. Padilla, "Liquid crystal tunable metamaterial absorber," Physical Review Letters, vol. 110, no. 17, article 177403, p. 2013.

[12] Q. Zhao, L. Kang, B. Du et al., "Electrically tunable negative permeability metamaterials based on nematic liquid crystals," Applied Physics Letters, vol. 90, no. 1, article 011112, 2007.

[13] T. Nesimoglu and C. Sabah, "A tunable metamaterial resonator using varactor diodes to facilitate the design of reconfigurable microwave circuits," IEEE Transactions on Circuits and Systems II: Express Briefs, vol. 63, no. 1, pp. 89-93, 2016.

[14] H. Yang, A. Kafaratzis, Z. Hu, N. Peng, and R. Gwilliam, "Ion implanted left-handed tunable metamaterial for microwave circuit applications," pp. 248-250, 2008.

[15] D. Wang, L. Ran, H. Chen, M. Mu, J. A. Kong, and B. I. $\mathrm{Wu}$, "Active left-handed material collaborated with microwave varactors," Applied Physics Letters, vol. 91, no. 16, pp. 164101-164101-3, 2007.

[16] H. Chen, B. I. Wu, L. Ran, T. M. Grzegorczyk, and J. A. Kong, "Controllable left-handed metamaterial and its application to a 
steerable antenna," Applied Physics Letters, vol. 89, no. 5, pp. 053509-053509-3, 2006.

[17] J. F. Wang, S. B. Qu, H. Ma, J. Zhang, and H. Du, "Tunable planar left-handed metamaterials based on split-ring resonator pairs," in 2015 IEEE MTT-S International Microwave Workshop Series on Advanced Materials and Processes for $R F$ and THz Applications (IMWS-AMP), pp. 1-3, Suzhou, China, July 2015.

[18] C. Sabah, "Tunable metamaterial design composed of triangular split ring resonator and wire strip for S-and C-microwave bands," Progress In Electromagnetics Research B, vol. 22, pp. 341-357, 2010.

[19] J. Wang, S. Qu, J. Zhang et al., “A tunable left-handed metamaterial based on modified broadside-coupled split-ring resonators," Progress In Electromagnetics Research Letters, vol. 6, pp. 35-45, 2009.

[20] J. Wang, S. Qu, Y. Yang, H. Ma, X. Wu, and Z. Xu, "Multiband left-handed metamaterials," Applied Physics Letters, vol. 95, no. 1, pp. 014105-014105-3, 2009.

[21] E. Ekmekci, K. Topalli, T. Akin, and G. Turhansayan, “A tunable multi-band metamaterial design using micro-split SRR structures," Optics Express, vol. 17, no. 18, pp. 16046-16058, 2009.

[22] H. Zhou, C. Wang, and H. Peng, "A novel double-incidence and multi-band left-handed metamaterials composed of double Z-shaped structure," Journal of Materials Science: Materials in Electronics, vol. 27, no. 3, pp. 2534-2544, 2016.

[23] A. Sarkhel, D. Mitra, and S. R. B. Chaudhuri, "A compact metamaterial with multi-band negative-index characteristics," Applied Physics A, vol. 122, no. 4, pp. 1-10, 2016.

[24] H. X. Xu, G. M. Wang, C. X. Zhang et al., "Multi-band left-handed metamaterial inspired by tree-shaped fractal geometry," Photonics and Nanostructures-Fundamentals and Applications, vol. 11, no. 1, pp. 15-28, 2013.

[25] O. Yurduseven, A. E. Yilmaz, and G. Turhan-Sayan, "Hybrid-shaped single-loop resonator: a four-band metamaterial structure," Electronics Letters, vol. 47, no. 25, pp. 13811382, 2011.

[26] H. Chen, L. Ran, J. Huangfu et al., "Metamaterial exhibiting left-handed properties over multiple frequency bands," Journal of Applied Physics, vol. 96, no. 9, pp. 5338-5340, 2004.

[27] O. Yurduseven, A. E. Yilmaz, and G. Turhan-Sayan, "Triangular-shaped single-loop resonator: a triple-band metamaterial with MNG and ENG regions in S/C bands," IEEE Antennas and Wireless Propagation Letters, vol. 10, pp. 701-704, 2011.

[28] J. Zhong, F. Wang, G. Wen et al., "Tunable triple-band negative permeability metamaterial consisting of single-loop resonators and ferrite," Journal of Electromagnetic Waves \& Applications, vol. 27, no. 3, pp. 267-275, 2013.

[29] X. Chen, T. M. Grzegorczyk, B. I. Wu, J. Pacheco, Jr., and J. A. Kong, "Robust method to retrieve the constitutive effective parameters of metamaterials," Physical Review E, vol. 70, 1 Part 2, pp. 811-811, 2004.

[30] J. B. Pendry, A. J. Holden, D. J. Robbins, and W. J. Stewart, "Magnetism from conductors and enhanced nonlinear phenomena," IEEE Transactions on Microwave Theory \& Techniques, vol. 47, no. 11, pp. 2075-2084, 1999.

[31] D. Ye, K. Chang, L. Ran, and H. Xin, "Microwave gain medium with negative refractive index," Nature Communications, vol. 5, article 5841, 2014.
[32] J. B. Pendry, A. J. Holden, W. J. Stewart, and I. I. Youngs, "Extremely low frequency plasmons in metallic mesostructures," Physical Review Letters, vol. 76, no. 25, pp. 47734776, 1996.

[33] D. R. Smith, S. Schultz, P. Markos, and C. M. Soukoulis, "Determination of effective permittivity and permeability of metamaterials from reflection and transmission coefficients," Physical Review B, vol. 65, no. 19, article 195104, 2001.

[34] H. Chen, J. Zhang, Y. Bai et al., "Experimental retrieval of the effective parameters of metamaterials based on a waveguide method," Optics Express, vol. 14, no. 26, pp. 12944-12949, 2006. 


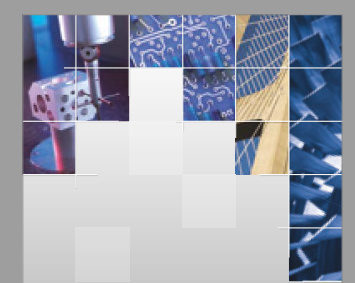

\section{Enfincering}
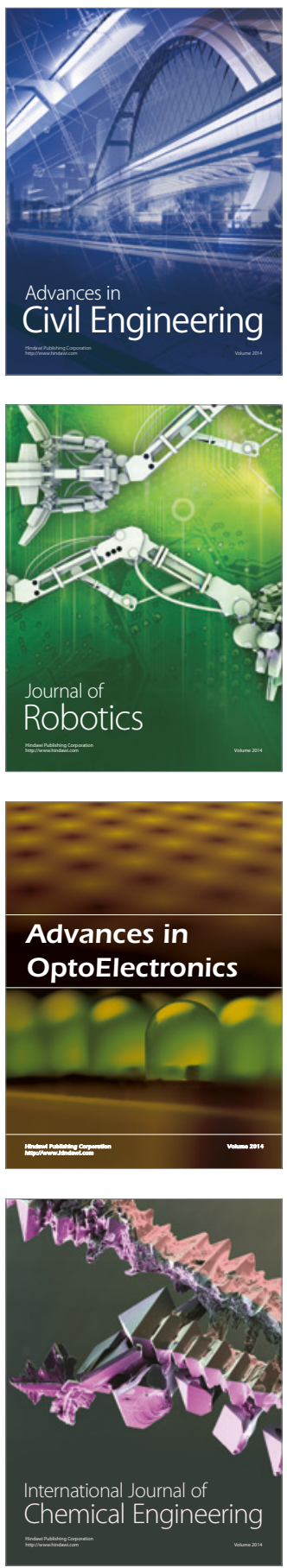

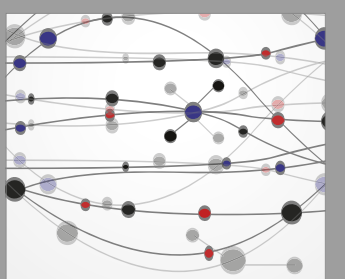

The Scientific World Journal

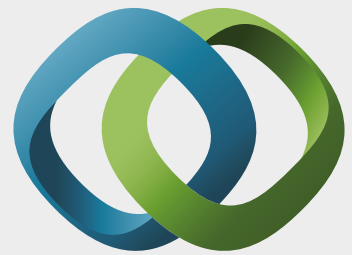

\section{Hindawi}

Submit your manuscripts at

https://www.hindawi.com
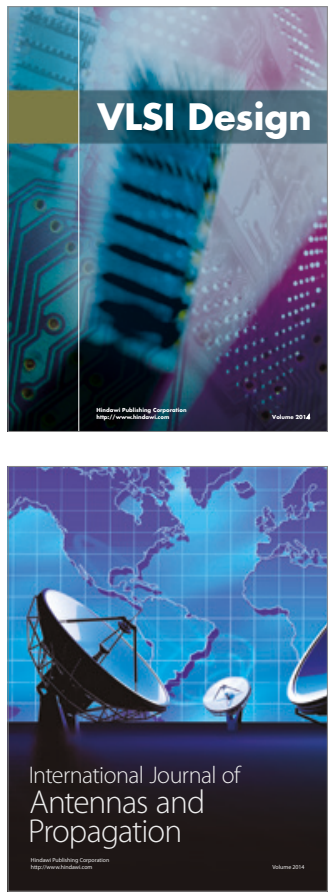

\section{Rotating}

Machinery
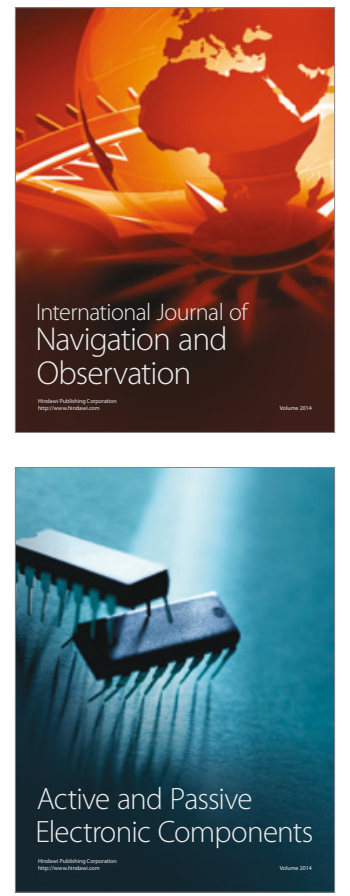
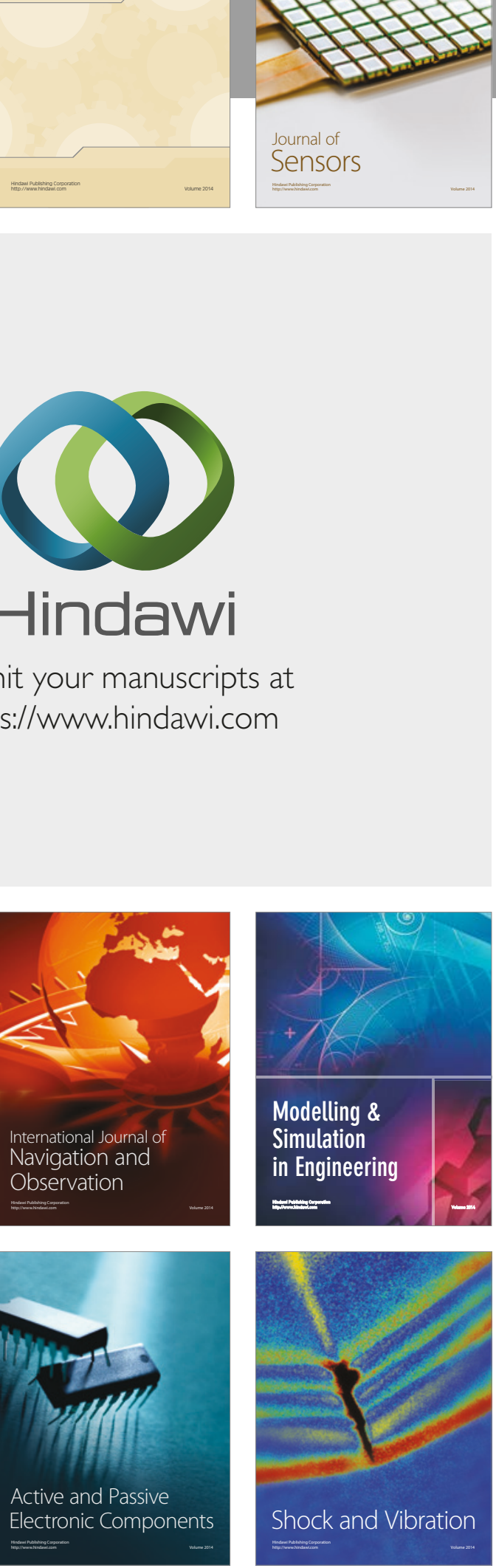
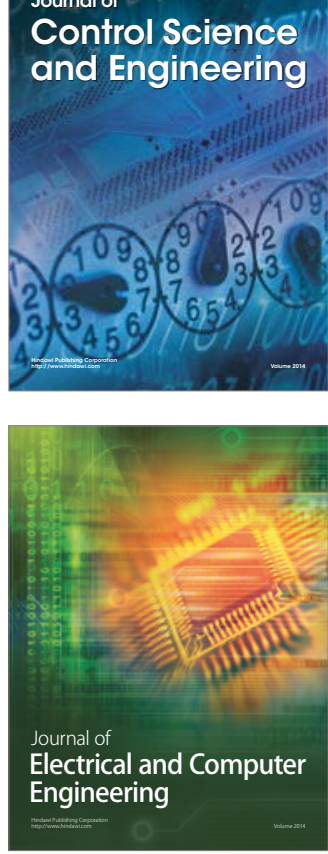

Distributed

Journal of

Control Science

and Engineering
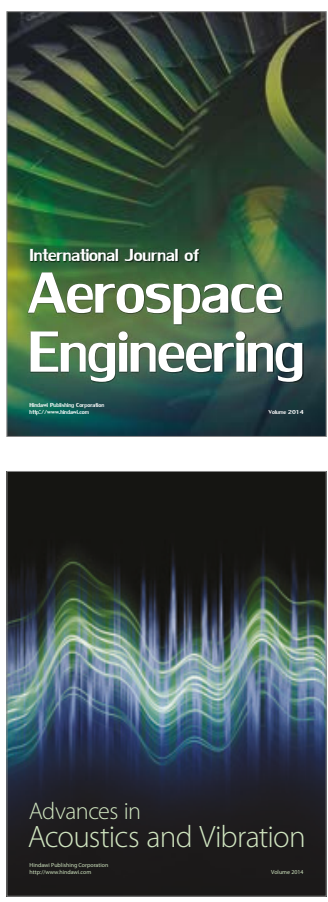

Sensor Networks 